\title{
Axisymmetric evolution of Einstein equations and mass conservation
}

\author{
Sergio Dain \\ Facultad de Matemática, Astronomía y Física, Universidad Nacional de Córdoba, Ciudad \\ Universitaria, (5000) Córdoba, Argentina \\ and \\ Max Planck Institute for Gravitational Physics (Albert Einstein Institute), Am Mühlenberg 1, \\ D-14476 Potsdam, Germany
}

Received 13 May 2008, in final form 10 June 2008

Published 30 June 2008

Online at stacks.iop.org/CQG/25/145021

\begin{abstract}
For axisymmetric evolution of isolated systems, we prove that there exists a gauge such that the total mass can be written as a positive definite integral on the spacelike hypersurfaces of the foliation and the integral is constant along the evolution. The conserved mass integral controls the square of the extrinsic curvature and the square of first derivatives of the intrinsic metric. We also discuss applications of this result for the global existence problem in axial symmetry.
\end{abstract}

PACS number: 04.20.Ex

\section{Introduction}

The main unsolved problem in general relativity is the global existence of solutions of Einstein's equations that describe the dynamics of strong gravitational fields. Symmetries have traditionally played an important role in this problem. The presence of a symmetry reduces the degrees of freedom of Einstein equations and hence considerably simplify its analysis. This is of course useful as a preliminary step in understanding the full problem but also many models with symmetries have direct physical applications.

In a vacuum, due to Birkhoff's theorem, spherical symmetry has no dynamics. For isolated systems, the next possible model with symmetries are axially symmetric spacetimes. It has been proved in [5] that no additional symmetry can be imposed to the spacetime if we want to keep the gravitational radiation and a complete null infinity. This result singles out axially symmetric spacetimes as the only models for an isolated, dynamical system with symmetries.

There exist many relevant physical models one can study in axial symmetry: headon collisions of two black holes, rotating starts and black holes, the critical collapse of gravitational waves. These models have been studied numerically. Particularly relevant for 
the results presented in this paper are the following references in which axial symmetry is imposed explicitly on the equations using cylindrical coordinates: [2, 8, 17, 23, 27-30].

From the analytical point of view, there exist no results up until now for axially symmetric isolated systems (see the review [25] for results with other kinds of symmetries in cosmologies). One of the purpose of this paper is to initiate the study of this problem. We mention a related problem: cosmologies with $U(1)$ symmetries. This symmetry was analyzed in [9, 12]. The equations are locally the same as in the axially symmetric case but the boundary conditions are radically different. In axial symmetry the Killing vector vanishes at the axis and that is the main source of the difficulties.

For axially symmetric data, the total ADM mass [1] can be written as a positive definite volume integral over one spacelike hypersurface (see [6, 14, 16, 19, 22]). This fact is likely to play a major role in the initial value (see the discussion in [15]). In order to study the implication of this integral formula for the evolution, the first natural step is to prove that there exists a gauge such that the mass integral holds not only at one hypersurface but in a whole foliation and it is conserved along the evolution. This is the subject of the present paper. To understand this result, let us review the notion of mass in general relativity and its conservation.

The total mass of an isolated system is a boundary integral at infinity calculated on a given spacelike hypersurface. If we consider not only one hypersurface but a foliation on the spacetime we can ask the question of whether the mass is conserved along this particular foliation. That is, whether the boundary integral gives the same result if it is calculated on different slices of the foliation. Any foliation is determined by the choice of a lapse function and a shift vector. If the lapse and shift of the foliation satisfy some fall-off conditions (this class is called asymptotically flat gauges), then the mass boundary integral is conserved. These notions appear naturally in the Hamiltonian formulation of general relativity (see [4, 24, 31, 32]).

In any physical theory conserved quantities (in particular, conserved energies) are very important to control the evolution of the system. However, in general relativity, the conserved mass appears as a boundary integral and not as a volume integral (as, for example, in the wave equation). Hence it is not possible to relate the mass with any norm of the fields to control their evolution (for the wave equation the energy is precisely the norm of the wave). Axially symmetric systems represent a remarkable exception. As was mentioned above in this case it is possible to write the mass as a positive definite volume integral. However, this integral formula is valid only in a particular gauge. Moreover, this gauge cannot be given a priori, it is a dynamical gauge which is prescribed as a solution of a system of differential equations. The natural question is whether this particular gauge satisfies the fall-off conditions which guarantee the conservation of the mass. Our main result is that the answer to that question is yes. In other words, there exists a gauge for which the mass can be written as a positive definite integral on each slice of the foliation and this integral is conserved along the evolution on this particular foliation. Then, in this gauge, the conserved mass controls the norm of the metric and the second fundamental form along the evolution and hence this particular gauge is likely to be the most relevant one to study axially symmetric isolated systems (we further discuss this point in section 6).

The plan of the paper is as follows. In section 2, we describe our main result which is given by theorem 2.1. In section 3 , we review the well-known $(2+1)+1$ formalism and compute the behavior of the fields at infinity and near the axis. In section 4 we describe the gauge. We also derive the mass integral formula in the $(2+1)+1$ formalism. This derivation is remarkably simpler than those presented so far in the literature. In section 5 we prove the main result. Finally, in section 6 we discuss the implications of this result for the evolution problem. 


\section{Main result}

An initial data set for the Einstein vacuum equations is given by a triple $\left(\bar{S}, \gamma_{a b}, K_{a b}\right)$ where $\bar{S}$ is a connected three-dimensional manifold, $\gamma_{a b}$ a (positive definite) Riemannian metric and $K_{a b}$ a symmetric tensor field on $\bar{S}$, such that the vacuum constraint equations

$$
\begin{aligned}
& \bar{D}_{b} K^{a b}-\bar{D}^{a} K=0, \\
& { }^{(3)} R-K_{a b} K^{a b}+K^{2}=0,
\end{aligned}
$$

are satisfied on $\bar{S}$, where $\bar{D}$ and ${ }^{(3)} R$ are the Levi-Civita connection and the Ricci scalar associated with $\gamma_{a b}$ and $K=K_{a b} \gamma^{a b}$. In these equations the indices are moved with the metric $\gamma_{a b}$ and its inverse $\gamma^{a b}$. For simplicity, in the present paper we assume $\bar{S}=\mathbb{R}^{3}$. We expect, however, that the results presented here generalize to the asymptotically Euclidean manifold with many ends.

We will further assume that the data are axially symmetric, which means that there exists a Killing vector field $\eta^{a}$, i.e

$$
£_{\eta} \gamma_{a b}=0,
$$

where $£$ denotes the Lie derivative, which has complete periodic orbits and such that

$$
£_{\eta} K_{a b}=0 .
$$

The Killing vector field has associated the following scalars with respect to the metric $\gamma_{a b}$. The norm

$$
\lambda^{2}=\gamma_{a b} \eta^{a} \eta^{b}
$$

and the twist

$$
\omega^{\prime}=\epsilon_{a b c} \eta^{a} \bar{D}^{b} \eta^{c}
$$

The prime in the notation of $\omega^{\prime}$ is justified in section 3, where we will show that $\omega^{\prime}$ is related to the time derivative of the four-dimensional twist potential $\omega$.

The data are called asymptotically flat if there exists a coordinate system $x^{\mu}(\mu=1,2,3)$ in $\mathbb{R}^{3}$ such that the metric components in these coordinates satisfy the fall-off conditions

$$
\gamma_{\mu \nu}=\delta_{\mu \nu}+o_{\infty}\left(r^{-1 / 2}\right)
$$

and the extrinsic curvature components

$$
K_{\mu \nu}=o_{\infty}\left(r^{-3 / 2}\right),
$$

where we write $f=o_{\infty}\left(r^{k}\right)$ if $f$ satisfies $\partial^{\alpha} f=o\left(r^{k-|\alpha|}\right)$, for all $\alpha$. Here $\delta_{\mu \nu}$ is the Euclidean metric in the coordinates $x^{\mu}, r$ is the Euclidean radius $r=\sqrt{x^{\mu} x^{\nu} \delta_{\mu \nu}}, \partial$ denotes partial derivatives with respect to the coordinates and $\alpha$ is a multindex.

The data are called maximal if the trace of $K_{a b}$ is equal to zero, i.e.

$$
K=\gamma^{a b} K_{a b}=0 .
$$

Following [18], we define the two-dimensional quotient metric $q_{a b}$ by

$$
q_{a b}=\gamma_{a b}-\frac{\eta_{a} \eta_{b}}{\lambda^{2}} .
$$

In [14] it is proved that for any axially symmetric and asymptotically flat metric $\gamma_{a b}$ there exists a coordinate system $(\rho, z, \phi)$, which we call the isothermal coordinates, such that the metric $q_{a b}$ and the Killing vector have the following form:

$$
q=\mathrm{e}^{2 u}\left(\mathrm{~d} \rho^{2}+\mathrm{d} z^{2}\right), \quad \eta^{a}=\left(\frac{\partial}{\partial \phi}\right)^{a},
$$


and the norm is given by

$$
\lambda=\rho \mathrm{e}^{\sigma / 2},
$$

where $u$ and $\sigma$ are smooth functions. To these coordinates we associated Cartesian coordinates $(x, y, z)$ via the standard formula $x=\rho \cos \phi, y=\rho \sin \phi$. It follows from the results proved in [14] that in the coordinates $(x, y, z)$ the metric $\gamma_{a b}$ is asymptotically flat. Hence, without loss of generality, in the following we take $x^{\mu}$ to be $(x, y, z)$.

In isothermal coordinates we consider the following integral:

$$
m=\frac{1}{16} \int_{-\infty}^{\infty} \mathrm{d} z \int_{0}^{\infty}\left[|\partial \sigma|^{2}+\frac{\mathrm{e}^{2 u} \omega^{2}}{\lambda^{4}}+2 \mathrm{e}^{2 u} K^{a b} K_{a b}\right] \rho \mathrm{d} \rho,
$$

where $|\partial \sigma|^{2}=\sigma_{, \rho}^{2}+\sigma_{, z}^{2}$. Note that the volume element $\rho \mathrm{d} z \mathrm{~d} \rho$ is equivalent to the volume element in Cartesian coordinates $\mathrm{d} x \mathrm{~d} y \mathrm{~d} z$ where the integration domain is $\mathbb{R}^{3}$. We will see in section 4 that for asymptotically flat, axially symmetric and maximal data $m$ defined by (13) is the total mass.

The mass integral (13) is positive definite, and is zero only for flat spacetime. The first two terms in the integrand contain the square of first derivatives of the metric and the third term contains the square of the extrinsic curvature.

We want to prescribe gauge conditions for the evolution such that the integral (13) holds not only at the initial surface but in any surface of the foliation. We prescribe the lapse by the requirement that the maximal condition (9) is preserved under evolution. The shift vector is prescribed by the condition that the isothermal coordinates are preserved by the evolution. In this way we obtain a coordinate system $(t, \rho, z, \phi)$, we call it the maximal-isothermal gauge. These gauge conditions are explicitly given in section 4 . This gauge has been used in numerical evolutions in the following references $[8,17,23,27,28]$. This is a dynamical gauge, namely, the lapse and shift are calculated in terms of the initial data at each step of the evolution.

The main result of this paper is given by the following theorem.

Theorem 2.1. Let $\left(\mathbb{R}^{3}, \gamma_{a b}, K_{a b}, \eta^{a}\right)$ be an axially symmetric, maximal, vacuum, asymptotically flat initial data. Then, there exists a unique solution of the maximal-isothermal gauge equations and the mass integral given by (13) is conserved along the evolution, that is

$$
\frac{\mathrm{d} m(t)}{\mathrm{d} t}=0 .
$$

This theorem is proved is section 4. We divide the proof in lemma 5.1, theorems 5.2 and 5.3. For the sake of clarity, in this section we presented the results using the standard $3+1$ decomposition. However, for axially symmetric spacetimes there exists a more natural decomposition, the $(2+1)+1$ formalism described in the section 3 . The maximal-isothermal gauge conditions and the mass formula are better expressed in this formalism.

\section{Axisymmetric evolution equations}

In this section we review the $(2+1)+1$ formalism, which has two parts. First, a reduction of the field equations by the action of the symmetry [18] and second a time plus space decomposition of the reduced equations (see $[8,23,27]$ ). We also give some useful formulae which relate the quantities in the $(2+1)+1$ formalism with the natural ones in the standard $3+1$ decomposition. 


\subsection{The $(2+1)+1$ formalism}

Consider a vacuum solution of Einstein's equations, i.e., a four-dimensional manifold $\mathcal{V}$ with metric $g_{a b}$ for which the Ricci tensor ${ }^{(4)} \mathcal{R}_{a b}$ vanishes. Suppose, in addition, that there exists a spacetime Killing vector $\eta^{a}$. We define the norm and the twist of $\eta^{a}$, respectively, by

$$
\lambda^{2}=\eta^{a} \eta^{b} g_{a b}, \quad \omega_{a}=\epsilon_{a b c d} \eta^{b} \bar{\nabla}^{c} \eta^{d},
$$

where $\bar{\nabla}_{a}$ is the connection and $\epsilon_{a b c d}$ the volume element with respect to $g_{a b}$. Assuming that the manifold is simply connected and using ${ }^{(4)} \mathcal{R}_{a b}=0$ it is possible to prove that $\omega_{a}$ is the gradient of a scalar field $\omega$

$$
\omega_{a}=\bar{\nabla}_{a} \omega \text {. }
$$

In our case the Killing field will be spacelike, i.e. $\lambda \geqslant 0$.

In the presence of a Killing field, there exists a well-known procedure to reduce the field equations [18]. Let $\mathcal{N}$ denote the collection of all trajectories of $\eta^{a}$, and assume that it is a differential 3-manifold. We define the Lorentzian metric $h_{a b}$ on $\mathcal{N}$ by

$$
g_{a b}=h_{a b}+\frac{\eta_{a} \eta_{b}}{\lambda^{2}}
$$

Einstein vacuum equation are equivalent to the following set of equations intrinsic to $\left(\mathcal{N}, h_{a b}\right)$

$$
\begin{aligned}
& \nabla_{a} \nabla^{a} \lambda=-\frac{1}{2 \lambda^{3}} \nabla^{a} \omega \nabla_{a} \omega, \\
& \nabla_{a} \nabla^{a} \omega=\frac{3}{\lambda} \nabla^{a} \omega \nabla_{a} \lambda, \\
& { }^{(3)} \mathcal{R}_{a b}=\frac{1}{\lambda} \nabla_{a} \nabla_{b} \lambda+\frac{1}{2 \lambda^{4}}\left(\nabla_{a} \omega \nabla_{b} \omega-\nabla_{c} \omega \nabla^{c} \omega h_{a b}\right),
\end{aligned}
$$

where $\nabla_{a}$ and ${ }^{(3)} \mathcal{R}_{a b}$ are the connexion and the Ricci tensor of $h_{a b}$. The system of equations (18)-(20) can be interpreted as the Einstein equation in three dimensions (equation (20)) coupled with a matter sources given by equations (18) and (19).

We make a $2+1$ decomposition of $\left(\mathcal{N}, h_{a b}\right)$ and the field equation (20). Let $n^{a}$ be the unit normal vector orthogonal to a spacelike, two-dimensional slice $S$. By construction we have $n^{a} \eta_{a}=0$. And hence the norm defined in (15) is equivalent to (5). The intrinsic metric on $S$ is denoted by $q_{a b}$ and it is given by

$$
h_{a b}=-n_{a} n_{b}+q_{a b} .
$$

Our convention for the signature of $h_{a b}$ is $(-++)$. In terms of the lapse $\alpha$ and shift vector $\beta^{a}$ the line element takes the form

$$
h=-\alpha^{2} \mathrm{~d} t^{2}+q_{i j}\left(\mathrm{~d} x^{i}+\beta^{i} \mathrm{~d} t\right)\left(\mathrm{d} x^{j}+\beta^{j} \mathrm{~d} t\right),
$$

where $i, j=1,2$. The extrinsic curvature $\chi_{a b}$ of the slices $S$ is given by 1

$$
\chi_{a b}=-q_{a}^{c} \nabla_{c} n_{b}=-\frac{1}{2} £_{n} q_{a b} .
$$

We write the field equations (20) as evolution equations for $\left(q_{a b}, \chi_{a b}\right)$

$$
\begin{aligned}
& \dot{q}_{a b}=-2 \alpha \chi_{a b}+£_{\beta} q_{a b}, \\
& \dot{\chi}_{a b}=£_{\beta} \chi_{a b}+\alpha\left(\chi \chi_{a b}+{ }^{(2)} R_{a b}-{ }^{(3)} \overline{\mathcal{R}}_{a b}-2 \chi_{a c} \chi_{b}^{c}\right)-D_{a} D_{b} \alpha,
\end{aligned}
$$

and constraint equations

1 Opposite sign convention with respect to [33]. 


$$
\begin{aligned}
& { }^{(2)} R-\chi^{a b} \chi_{a b}+\chi^{2}=\mu, \\
& D^{a} \chi_{a b}-D_{b} \chi=J_{b},
\end{aligned}
$$

where a dot denotes partial derivative with respect to the $t$ coordinate, ${ }^{(2)} R_{a b}$ is the Ricci tensor and $D_{a}$ is the connexion with respect to $q_{a b},{ }^{(3)} \overline{\mathcal{R}}_{a b}$ denotes the projection of ${ }^{(3)} \mathcal{R}_{a b}$ into $S$ and

$$
\begin{aligned}
& \mu=2^{(3)} \mathcal{R}_{a b} n^{a} n^{b}+{ }^{(3)} \mathcal{R}, \\
& J_{b}=-q_{b}^{c} n^{a(3)} \mathcal{R}_{c a} .
\end{aligned}
$$

The scalar $\mu$, which appears as 'matter source' in the Hamiltonian constraint (28), will be relevant in the computation of the mass. We will need its explicit expression

$$
\mu=2 \frac{|D \lambda|^{2}}{\lambda^{2}}+2 D_{a}\left(\frac{D^{a} \lambda}{\lambda}\right)+2 \frac{\lambda^{\prime}}{\lambda} \chi+\frac{1}{2 \lambda^{4}}\left(\omega^{\prime 2}+|D \omega|^{2}\right),
$$

where the prime denotes directional derivative with respect to $n^{a}$, that is

$$
\lambda^{\prime}=n^{a} \nabla_{a} \lambda=\mathfrak{£}_{n} \lambda .
$$

Note that

$$
\lambda^{\prime}=\alpha^{-1}\left(\dot{\lambda}-\mathfrak{f}_{\beta} \lambda\right) .
$$

To obtain equation (30) we have used equations (18) and (19).

Finally, it is convenient to decompose the extrinsic curvature $\chi_{a b}$ in its trace $\chi$ and trace free part $k_{a b}$, that is

$$
k_{a b}=\chi_{a b}-q_{a b} \frac{\chi}{2} .
$$

\subsection{Relations with the $3+1$ decomposition}

Take the definition of the spacetime twist potential (15) and (16). The derivative $\omega^{\prime}$ is given by

$$
\omega^{\prime}=n^{a} \bar{\nabla}_{a} \omega=n^{a} \epsilon_{a b c d} \eta^{b} \bar{\nabla}^{c} \eta^{d} .
$$

Note that $n^{a} \epsilon_{a b c d}$ is the volume element on the three-dimensional slice $\bar{S}$ denoted by $\epsilon_{a b c}$ in section 2. The covariant derivative $\bar{\nabla}_{a}$ in (34) appears antisymmetrized and hence we can replace it by any covariant derivative, in particular $\bar{D}_{a}$. Then, we obtain that (34) is equivalent to (6).

The twist potential $\omega$ can be calculated in terms of the initial data as follows. Define the vector $S^{a}$ by

$$
S_{a}=K_{a b} \eta^{b} .
$$

Using the definition of $K_{a b}$

$$
K_{a b}=-\gamma_{a}^{c} \bar{\nabla}_{c} n_{b},
$$

we obtain

$$
S_{a}=-n^{c} \bar{\nabla}_{c} \eta_{a},
$$

where we have used the Killing equation $\bar{\nabla}_{(a} \eta_{b)}=0$, equation $\eta_{a} n^{a}=0$ and the following expression for the metric $\gamma_{a b}$ :

$$
g_{a b}=\gamma_{a b}-n_{a} n_{b} .
$$

6 
We use the following expression for the covariant derivative of $\eta_{a}$ (see [18]):

$$
\bar{\nabla}_{a} \eta_{b}=\frac{1}{2 \lambda^{2}} \epsilon_{a b c d} \eta^{c} \omega^{d}+\frac{1}{2 \lambda} \eta_{[a} \bar{D}_{b]} \lambda .
$$

Using (39) and (37) we obtain

$$
\epsilon_{a b c} S^{b} \eta^{c}=-\frac{1}{2} \bar{D}_{a} \omega
$$

Note that the left-hand side of equation (40) is calculated only with the initial data. This is the desired equation.

The relation between the trace of the extrinsic curvature $K$ of the slice $\bar{S} d$ and the trace of extrinsic curvature $\chi$ of the slice $S$ is given by

$$
K=\chi-\frac{\lambda^{\prime}}{\lambda} .
$$

If we prescribe $K=0$ then we have

$$
\chi=\frac{\lambda^{\prime}}{\lambda} \text {. }
$$

The relation between the two extrinsic curvatures is given by

$$
\chi_{a b}=q_{a}^{c} q_{b}^{d} K_{c d} .
$$

The following component of the extrinsic curvature will be used in the next section:

$$
K_{\phi \phi}=K_{a b} \eta^{a} \eta^{b}=-\lambda^{\prime} \lambda .
$$

Using equations (35), (40), (43) and (44) we obtain the following expression for the square of $K_{a b}$ :

$$
K^{a b} K_{a b}=\chi^{a b} \chi_{a b}+\frac{\lambda^{\prime 2}}{\lambda^{2}}+\frac{|D \omega|^{2}}{2 \lambda^{4}} .
$$

\section{Maximal-isothermal gauge and mass integral}

In this section, we review the well-known maximal-isothermal gauge (see $[8,17,23,27,28]$ ) using the $(2+1)+1$ formalism presented in the previous section. We analyze the fall-off behavior of the fields in this gauge near the axis at infinity. Finally, we present a new derivation of the mass integral formula.

\subsection{Gauge}

The maximal gauge condition is well known. In the standard $3+1$ decomposition, the equation for the lapse is given by

$$
\Delta_{\gamma} \alpha=\alpha K^{a b} K_{a b},
$$

where $\Delta_{\gamma}$ denotes the Laplacian with respect to the metric $\gamma_{a b}$.

The shift vector is fixed by the requirement that the isothermal coordinates are preserved under the evolution. In isothermal coordinates, the two-dimensional metric $q_{a b}$ has the form (11). If we take a time derivative to (11) we obtain

$$
\dot{q}_{a b}=2 \dot{u} q_{a b},
$$

that is, the tensor $\dot{q}_{a b}$ is pure trace. Hence, the trace free part (with respect to $q_{a b}$ ) of the evolution equation (24) is given by

$$
\left(\mathcal{L}_{q} \beta\right)_{a b}=2 \alpha k_{a b},
$$


where $\mathcal{L}_{q}$ is the conformal Killing operator with respect to $q_{a b}$

$$
\left(\mathcal{L}_{q} \beta\right)_{a b}=D_{a} \beta_{b}+D_{b} \beta_{a}-q_{a b} D_{c} \beta^{c} .
$$

Equation (48) constitute a first-order elliptic system which determines $\beta^{a}$ under appropriate boundary conditions, as we will see in section 5. Summarizing, the lapse and shift for the maximal-isothermal gauge are determined by equations (46) and (48).

\subsection{Fall-off behavior and axial regularity in isothermal coordinates}

The fall of conditions (7) for the three-dimensional metric implies that (see [14])

$$
u=\sigma=o_{\infty}\left(r^{-1 / 2}\right) .
$$

From equations (43) and equation (8) we obtain

$$
\chi_{i j}=o_{\infty}\left(r^{-3 / 2}\right) .
$$

The function $\lambda^{\prime} / \lambda$ will be important in the following section, we need to compute its fall-off behavior. This behavior is not an obvious consequence of (8) because the relation between these two functions involves a quotient by $\rho^{2}$. To compute the fall off of this function we proceed as follows. In Cartesian components we have

$$
\eta^{x}=-y, \quad \eta^{y}=x
$$

and hence

$$
K_{\phi \phi}=y^{2} K_{x x}-2 x y K_{x y}+x^{2} K_{y y} .
$$

Following [14] it is enough to consider the plane $x=0$ which is transversal to the Killing vector. If we use (44), (53) and (12) and then evaluate at $x=0$ we have

$$
-\frac{\lambda^{\prime}}{\lambda}=\frac{K_{\phi \phi}}{\lambda^{2}}=\mathrm{e}^{-\sigma} K_{x x}
$$

Hence, at this plane the function $\lambda^{\prime} / \lambda$ is smooth and it has the same fall off as $K_{a b}$, namely

$$
\frac{\lambda^{\prime}}{\lambda}=o_{\infty}\left(r^{-3 / 2}\right)
$$

Since the plane $x=0$ is transversal to the axial Killing vector it follows that (55) holds everywhere.

The regularity of the metric $\gamma_{a b}$ and the extrinsic curvature $K_{a b}$ at the axis implies restrictions on the behavior of the different quantities (for a detailed discussion of this issue see [27] and [29]). We summarize here two important consequences of axial regularity. First, the function $q$ defined by

$$
q=u-\frac{\sigma}{2}
$$

vanished at the axis (see [14])

$$
q(\rho=0)=0 .
$$

Second, the functions involved satisfy parity conditions with respect to the $\rho$ coordinate. In particular we have

$$
u, q, \sigma, k_{\rho}^{\rho} \text { are even functions of } \rho,
$$

and

$$
\lambda, k_{z}^{\rho} \text { are odd functions of } \rho .
$$

Note that odd functions vanish at the axis. 


\subsection{Mass formula}

Consider the Hamiltonian constraint (26). Using equations (12) and (56) we write the Hamiltonian constraint in terms of $\rho, \sigma$ and $q$ in such a way that there are no singular terms at the axis. To do this, we use the following elementary identities.

The Ricci scalar of $q_{a b}$ is given by

$$
{ }^{(2)} R=-2 \mathrm{e}^{-2 u} \Delta u \text {, }
$$

where $\Delta$ is the flat two-dimensional Laplacian

$$
\Delta u=u_{, \rho \rho}+u_{, z z}
$$

Using equation (12) we obtain

$$
\frac{|D \lambda|^{2}}{\lambda^{2}}=|D \log \lambda|^{2}=\left|D\left(\frac{\sigma}{2}+\log \rho\right)\right|^{2} .
$$

Finally, the $\rho$ coordinate satisfy

$$
\text { (3) } \Delta \log \rho=0 \text {, }
$$

where we have defined ${ }^{(3)} \Delta$ as the three-dimensional flat Laplacian

$$
{ }^{(3)} \Delta u=\Delta u+\frac{u_{, \rho}}{\rho} \text {. }
$$

Using equations (56), (60), (62) and (63) the Hamiltonian constraint (26) can be written in the following form:

$-2^{(3)} \Delta \sigma-2 \Delta q=\frac{\mathrm{e}^{2 u}}{2}\left[2 \chi^{a b} \chi_{a b}+2 \chi\left(-\chi+2 \frac{\lambda^{\prime}}{\lambda}\right)+|D \sigma|^{2}+\frac{1}{\lambda^{4}}\left(\omega^{\prime 2}+|D \omega|^{2}\right)\right]$.

We define the mass as the integral of the right-hand side of this equation, namely

$$
m=\frac{1}{16} \int_{S}\left(2 k^{a b} k_{a b}-\chi^{2}+4 \chi \frac{\lambda^{\prime}}{\lambda}+|D \sigma|^{2}+\frac{1}{\lambda^{4}}\left(\omega^{\prime 2}+|D \omega|^{2}\right)\right) \rho \mathrm{d} V_{q},
$$

where we have used (33) and $\mathrm{d} V_{q}=\mathrm{e}^{2 u} \mathrm{~d} \rho \mathrm{d} z$ denotes the volume element with respect to $q_{a b}$. Note that we introduce a weight $\rho$ in the integral. If we impose the condition $K=0$ (using equation (41)) we get that the integrand is positive definite, namely

$$
m=\frac{1}{16} \int_{S}\left(2 k^{a b} k_{a b}+3 \frac{\lambda^{\prime 2}}{\lambda^{2}}+|D \sigma|^{2}+\frac{1}{\lambda^{4}}\left(\omega^{\prime 2}+|D \omega|^{2}\right)\right) \rho \mathrm{d} V_{q}
$$

For maximal two-dimensional slices $\chi=0$ the integral (66) is also positive definite. However, this choice will not give an equation for the lapse function (analog to (46)) with positive definite solutions. To prove the equivalence of equation (67) with (13) we use (45).

The crucial point is that this volume integral can be written as a boundary integral at infinity using the left-hand side of equation (65). We have

$$
\left.m=-\frac{1}{4} \int_{-\infty}^{\infty} \mathrm{d} z \int_{0}^{\infty}{ }^{(3)} \Delta \sigma+\Delta q\right) \rho \mathrm{d} \rho .
$$

This integral can be converted into a boundary integral at infinity as follows. For the first term we use that $\rho \mathrm{d} \rho \mathrm{d} z$ is the volume element in $\mathbb{R}^{3}$ and then we can use the divergence theorem in three dimensions to obtain

$$
\int_{-\infty}^{\infty} \mathrm{d} z \int_{0}^{\infty}{ }^{(3)} \Delta \sigma \rho \mathrm{d} \rho \mathrm{d} z=\lim _{r \rightarrow \infty} \int_{0}^{\pi} \partial_{r} \sigma r \rho \mathrm{d} \theta .
$$

The second term in (68) can be also written in the divergence form

$$
\int \mathrm{d} \rho \mathrm{d} z\left(q_{, \rho \rho}+q_{, z z}\right) \rho=\int \mathrm{d} \rho \mathrm{d} z\left(\left(\rho q_{, \rho}-q\right)_{, \rho}+\left(\rho q_{, z}\right)_{, z}\right) .
$$


We use the divergence theorem in two dimensions to transform this volume integral in a boundary integral. Namely, let $\Omega$ be an arbitrary domain, we have

$$
\int_{\Omega} \mathrm{d} \rho \mathrm{d} z\left(\left(\rho q_{, \rho}-q\right)_{, \rho}+\left(\rho q_{, z}\right)_{, z}\right)=\oint_{\partial \Omega} \bar{V} \cdot \bar{n} \mathrm{~d} \bar{s},
$$

where $\bar{n}$ is the two-dimensional unit normal, $\mathrm{d} \bar{s}$ the line element of the one-dimensional boundary $\partial \Omega$ and $\bar{V}$ is the two-dimensional vector given in coordinates $(\rho, z)$ by

$$
\bar{V}=\left(\left(\rho q_{, \rho}-q\right),\left(\rho q_{, z}\right)\right) \text {. }
$$

Let $\Omega$ be the half plane $\rho \geqslant 0$. By (57) and the assumption that $q$ is smooth we have that the vector $\bar{V}$ vanishes at the axis. Then the only contribution of the boundary integral is at infinity, namely

$$
\int_{\rho \geqslant 0} \Delta_{2} q \rho \mathrm{d} \rho \mathrm{d} z=\lim _{r \rightarrow \infty} \int_{0}^{\pi}\left(r \partial_{r} q-q\right) \rho \mathrm{d} \theta .
$$

Summing (73) and (69) we obtain the final expression for the mass as boundary integral

$$
m=-\frac{1}{4} \lim _{r \rightarrow \infty} \int_{0}^{\pi}\left(\partial_{r} \sigma+\partial_{r} q-\frac{q}{r}\right) r \rho \mathrm{d} \theta .
$$

We can write this expression in terms of $u$ instead of $q$

$$
m=-\frac{1}{4} \lim _{r \rightarrow \infty} \int_{0}^{\pi}\left(\frac{1}{2} \partial_{r} \sigma+\frac{\sigma}{2 r}+\partial_{r} u-\frac{u}{r}\right) r \rho \mathrm{d} \theta
$$

Until now we have not mentioned the relation of $m$ with the total mass. In fact in order to prove theorem 2.1 we do not need any other information. The quantity $m$ is a positive definite integral that can be expressed as a boundary integral at infinity. As a consequence we will prove that it is conserved along the evolution in the maximal-isothermal gauge.

However, it is possible to show that $m$ is precisely the total mass of the spacetime. There is an interesting and subtle point here. The equivalence of this formula with the total mass have been previously proved using a strong fall-off condition for $q$ (see $[6,16,19]$ ), namely

$$
q=o_{\infty}\left(r^{-3 / 2}\right)
$$

which implies that $q$ does not appear in the boundary integral (75). Recently, this equivalence was proved without this restriction [14]. In the deduction we have made, we have not used any restriction for $q$ which is consistent with [14]. Note the simplicity of this deduction (which is much in the spirit of the energy defined in [12] for cosmologies) compared with those using three-dimensional slices like [19].

We also mention that in the following section we will prove that the strong decay condition (76) is preserved by the evolution.

\section{Existence of the gauge and mass conservation}

We begin with the lapse equation (46). This condition does not require any symmetry on the data. The existence and uniqueness of the solution of this equation in the standard $3+1$ decomposition are well known. For completeness we review this result.

Lemma 5.1. There exists a unique smooth strictly positive solution $\alpha$ of equation (46) with the following fall-off

$$
\alpha-1=o_{\infty}\left(r^{-1 / 2}\right) .
$$


Proof. Set

$$
\bar{\alpha}=\alpha-1,
$$

then, using (46), we have that

$$
\Delta_{\gamma} \bar{\alpha}-f \bar{\alpha}=f
$$

where $f=K^{a b} K_{a b} \geqslant 0$. The fall-off behavior assumed for $K_{a b}$ in (8) implies that $f \in H_{-3 / 2}^{s}$ for all $s$. Here $H_{\delta}^{s}$ denote weighted Sobolev spaces. See [3, 7, 10] and reference therein for definition and properties of these spaces. We use the index notation of [3].

The decay assumed on the metric $\gamma_{a b}$ (7) implies that the operator $\Delta_{\gamma}-f: H_{s+2, \delta} \rightarrow$ $H_{s, \delta-2}$ is an isomorphism for $\delta=-1 / 2$ (see for example [11,21]). Note that we use this theorem for dimension 3. Then there exists a unique solution $\bar{\alpha} \in H_{-1 / 2}^{s}$ of equation (79). Moreover $\bar{\alpha} \geqslant 0$ by the maximum principle (for a version of the maximum principle for non-compact manifold see for example [11]). Hence, we have that $\alpha>0$.

If the data is axially symmetric then the solution $\alpha$ will be also axially symmetric: take the Lie derivative with respect to $\eta^{a}$ to both sides of equation (79), we get

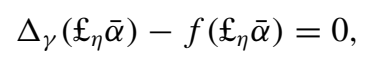

since $£_{\eta} \bar{\alpha} \in H_{-1 / 2}^{s}$ (to see this, take the expression of $\eta$ in Cartesian coordinates (52)), the isomorphism theorem mentioned above implies that $£_{\eta} \bar{\alpha}=0$. Finally, we note that since $\alpha$ is smooth in $(x, y, z)$, it follows that an axially symmetric $\alpha$ is an even function of $\rho$.

We consider now the equation for the shift. Instead of solving the first-order system (48) we will solve a second-order system obtained from taking a derivative to (48). Then, we will show that a solution of the second-order system is also a solution of the original equation (48) under appropriate boundary conditions.

Under the conformal transformations (11), the conformal Killing operator rescale like

$$
\mathcal{L}_{q}(\beta)_{a b}=\mathrm{e}^{2 u} \mathcal{L}\left(\mathrm{e}^{-2 u} \beta\right)_{a b},
$$

where $\mathcal{L}$ is the flat conformal Killing operator

$$
(\mathcal{L} \beta)_{a b}=\partial_{a} \beta_{b}+\partial_{b} \beta_{a}-q_{a b} \partial_{c} \beta^{c} .
$$

We take a flat divergence to equation (48) and use equation (81) to get

$$
\Delta\left(\mathrm{e}^{-2 u} \beta_{a}\right)=2 \partial^{b}\left(\alpha \mathrm{e}^{-2 u} k_{a b}\right),
$$

where we have used

$$
\partial^{b}(\mathcal{L} \beta)_{a b}=\Delta \beta_{a} .
$$

If we contract equation (83) with $\delta^{a b}$ and use $q^{a b}=\mathrm{e}^{-2 u} \delta^{a b}$ we obtain our final equation

$$
\Delta \beta^{a}=2 \partial^{b}\left(\alpha k_{b}^{a}\right)
$$

where $\beta^{a}=q^{a b} \beta_{b}$ and $k_{b}^{a}=q^{a c} k_{c b}$.

As was mentioned in section 4.2, the smoothness of the metric at the axis implies that the relevant functions satisfy parity conditions in the $\rho$ coordinate. In particular, the components of the shift vector $\beta^{a}$ should satisfy

$$
\beta^{z}(\rho, z)=\beta^{z}(-\rho, z), \quad \beta^{\rho}(\rho, z)=-\beta^{\rho}(-\rho, z) .
$$

Theorem 5.2. There exists a unique, smooth, solution $\beta^{a}$ of equation (85) with the following fall-off behavior

$$
\beta^{a}=o_{\infty}\left(r^{-1 / 2}\right)
$$

The solution satisfies the parity conditions (86) and the gauge equation (48). 
Moreover, the quotient $\beta^{\rho} / \rho$ is smooth and have the following fall-off

$$
\frac{\beta^{\rho}}{\rho}=o_{\infty}\left(r^{-3 / 2}\right)
$$

Remark: the fall-off condition (88) will be essential in the proof of the mass conservation theorem 5.3.

Proof. We distinguish the two components of equation (85)

$$
\Delta \beta^{\rho}=F^{\rho}, \quad \Delta \beta^{z}=F^{z},
$$

where

$$
F^{\rho}=2 \partial^{j}\left(\alpha k_{j}^{\rho}\right), \quad F^{z}=2 \partial^{j}\left(\alpha k_{j}^{z}\right) .
$$

The function $\alpha$ is even in $\rho$, using (58) and (59) we obtain that $F^{\rho}$ is odd and $F^{z}$ is even in $\rho$.

In terms of the cylindrical coordinates $(\rho, z)$, the source functions $F$ are defined only for $\rho \geqslant 0$. But because they satisfy parity conditions we can smoothly extend them to all $\mathbb{R}^{2}$ by the following prescription:

$$
F^{\rho}(\rho, z)=-F^{\rho}(-\rho, z), \quad F^{z}(\rho, z)=F^{z}(-\rho, z) .
$$

Hence, we can consider equations (89) as Poisson equations

$$
\Delta u=f,
$$

in $\mathbb{R}^{2}$ with decay conditions at infinity (87). Uniqueness of the solution of (92) under the decay condition (87) follows immediately integrating by parts the Laplace equation. Namely, for any domain $\Omega$ we have

$$
\int_{\Omega} u \Delta u=\int_{\Omega} \partial(u \partial u)-|\partial u|^{2}=\oint_{\partial \Omega} u \partial_{n} u-\int_{\Omega}|\partial u|^{2},
$$

and hence for a solution of $\Delta u=0$ we have

$$
\oint_{\partial \Omega} u \partial_{n} u=\int_{\Omega}|\partial u|^{2}
$$

Let $\Omega$ be a ball of radius $R$, and take the limit $R \rightarrow \infty$. By the decay condition (87), the boundary integral in (94) vanishes. Then, it follows that $u$ must be constant, by the decay condition it must be zero. Since the homogeneous equation has only the trivial solution uniqueness follows. Also, for solutions in this class, integrating equation (92) we obtain that the source must satisfy the condition

$$
\int_{\mathbb{R}^{2}} f=0
$$

We need to verify (95) for our source functions. The function $F^{\rho}$ is odd in $\rho$, hence it satisfies (95) automatically. For $F^{z}$ we use that it can be written in the divergence form

$$
F^{z}=\partial_{i} v^{i}, \quad v^{i}=2 \alpha k_{z}^{i} .
$$

Then we have

$$
\int_{\mathbb{R}^{2}} F^{z}=2 \int_{\rho \geqslant 0} F^{z}=2 \int_{\rho \geqslant 0} \partial_{i} v^{i}
$$

using the divergence theorem in two dimension we get

$$
\int_{\rho \geqslant 0} \partial_{i} v^{i}=\left.\int_{0}^{\infty} v^{\rho}\right|_{\rho=0} \mathrm{~d} \rho+\lim _{R \rightarrow \infty} \oint_{C_{R}} v^{r} \mathrm{~d} s,
$$


where $C_{R}$ denotes the semicircle with $r=R$. By the decay condition (51) for $k_{a b}$ and (77) for the lapse, we have that the second boundary integral vanishes. For the first one, we use equation (59) to conclude that

$$
\left.v^{\rho}\right|_{\rho=0}=0 \text {. }
$$

And hence we obtain the desired result that $F^{z}$ satisfies (95).

To actually obtain a solution of equation (92) we use the Green formula for the Laplacian in two dimensions $G\left(x, x^{\prime}\right)=-\log \left|x-x^{\prime}\right|^{2}$. A solution $u$ has the integral representation

$$
u(x)=\int_{\mathbb{R}^{2}} G\left(x, x^{\prime}\right) f\left(x^{\prime}\right) \mathrm{d} x^{\prime} .
$$

In polar coordinates $(r, \theta)$ (related to $(\rho, z)$ by the standard formula $z=r \cos \theta \rho=r \sin \theta$ ) we have the well-known expansion of $G$

$$
\begin{aligned}
G\left(r, \theta ; r^{\prime}, \theta^{\prime}\right) & =-2 \log r+2 \sum_{n=1}^{\infty} \frac{r^{\prime n}}{n r^{n}} \cos \left(n\left(\theta-\theta^{\prime}\right)\right) \quad \text { for } \quad r>r^{\prime} \\
& =-2 \log r^{\prime}+2 \sum_{n=1}^{\infty} \frac{r^{n}}{n r^{\prime n}} \cos \left(n\left(\theta-\theta^{\prime}\right)\right) \quad \text { for } \quad r<r^{\prime}
\end{aligned}
$$

We use the trigonometric identity

$$
\cos \left(n\left(\theta-\theta^{\prime}\right)\right)=\cos (n \theta) \cos \left(n \theta^{\prime}\right)+\sin (n \theta) \sin \left(n \theta^{\prime}\right),
$$

to split the sum in terms which are odd functions of $\theta$ (and hence of $\rho$ ) and terms which are even functions of $\theta$.

If the source function $f$ in the integral (100) is odd in $\rho$, then the terms involving $\cos \left(n \theta^{\prime}\right)$ vanish, for even source functions the terms with $\sin \left(n \theta^{\prime}\right)$ vanish.

We are interested in the fall-off behavior of the solution. It is convenient to split the source functions $F$ into two terms, one with compact support in some ball and the other which pick up the decay behavior. Let $\chi: \mathbb{R} \rightarrow \mathbb{R}$ be a cut-off function such that $\chi \in C^{\infty}(\mathbb{R}), 0 \leqslant \chi \leqslant 1, \chi(t)=1$ for $0 \leqslant t \leqslant 1, \chi(t)=0$ for $2 \leqslant t$ and write $\chi_{R}(r)=\chi(r / R), f_{\infty}=\left(1-\chi_{R}\right) f$, where $R$ is an arbitrary positive number.

Using the expansion (101) we compute the integral (100). Note that the term with $\log r$ vanishes due to (95). For $r>R$ we have for $\beta^{\rho}$

$$
\left.\beta^{\rho}=2 \sum_{n=1}^{\infty} \frac{\sin (n \theta)}{n}\left[r^{-n} A_{n}+B_{n}(r)+C_{n}(r)\right)\right],
$$

where

$$
\begin{aligned}
& A_{n}=\int_{0}^{R} r^{\prime n} \sin \left(n \theta^{\prime}\right) F^{\rho} r^{\prime} \mathrm{d} r^{\prime} \mathrm{d} \theta^{\prime}, \\
& B_{n}(r)=r^{-n} \int_{R}^{r} r^{\prime n} \sin \left(n \theta^{\prime}\right) F_{\infty}^{\rho} r^{\prime} \mathrm{d} r^{\prime} \mathrm{d} \theta^{\prime}, \\
& C_{n}(r)=r^{n} \int_{r}^{\infty} \frac{1}{r^{\prime n}} \sin \left(n \theta^{\prime}\right) F_{\infty}^{\rho} r^{\prime} \mathrm{d} r^{\prime} \mathrm{d} \theta^{\prime}
\end{aligned}
$$

For $\beta^{z}$ we have a similar expansion for $r>R$

$$
\left.\beta^{z}=2 \sum_{n=1}^{\infty} \frac{\cos (n \theta)}{n}\left[r^{-n} A_{n}+B_{n}(r)+C_{n}(r)\right)\right],
$$


where

$$
\begin{aligned}
& A_{n}=\int_{0}^{R} r^{\prime n} \cos \left(n \theta^{\prime}\right) F^{z} r^{\prime} \mathrm{d} r^{\prime} \mathrm{d} \theta^{\prime}, \\
& B_{n}(r)=r^{-n} \int_{R}^{r} r^{\prime n} \cos \left(n \theta^{\prime}\right) F_{\infty}^{z} r^{\prime} \mathrm{d} r^{\prime} \mathrm{d} \theta^{\prime}, \\
& C_{n}(r)=r^{n} \int_{r}^{\infty} \frac{1}{r^{\prime n}} \cos \left(n \theta^{\prime}\right) F_{\infty}^{z} r^{\prime} \mathrm{d} r^{\prime} \mathrm{d} \theta^{\prime}
\end{aligned}
$$

We use the decay condition (51) to obtain that the sources satisfy

$$
F^{\rho}=F^{z}=o_{\infty}\left(r^{-5 / 2}\right) .
$$

From (112) we get that

$$
B_{n}(r)=C_{n}(r)=o_{\infty}\left(r^{-1 / 2}\right) .
$$

These are the solutions of our problem. It is clear that they satisfy the parity conditions (86).

The important property of the representation (104) is that it allows us to prove (88). In effect, we use the trigonometric identity [20]

$$
\sin (n \theta)=\sum_{k=0}^{n-1}\left(\begin{array}{l}
n \\
k
\end{array}\right)(\cos \theta)^{k}(\sin \theta)^{n-k} \sin \left(\frac{1}{2}(n-k) \pi\right)
$$

to obtain that the function $\Theta_{n}$ defined by

$$
\Theta_{n}(\theta)=\frac{\sin (n \theta)}{\sin \theta}=\sum_{k=0}^{n-1}\left(\begin{array}{l}
n \\
k
\end{array}\right)(\cos \theta)^{k}(\sin \theta)^{n-k-1} \sin \left(\frac{1}{2}(n-k) \pi\right)
$$

is a smooth function. Hence, using (104), we obtain the expansion

$$
\left.\frac{\beta^{\rho}}{\rho}=2 \sum_{n=1}^{\infty} \frac{\Theta_{n}(\theta)}{n}\left[r^{-n+1} A_{n}+r^{-1} B_{n}(r)+r^{-1} C_{n}(r)\right)\right] .
$$

Using a similar argument as above we obtain (88).

Finally, we need to verify that our solution of equation (85) is also a solution of the first-order system (48). Define the trace free tensor $t_{a b}$ by

$$
t_{a b}=\left(\left(\mathcal{L}_{q} \beta\right)_{a b}-2 \alpha k_{a b}\right) \mathrm{e}^{-2 u} .
$$

We have proved that

$$
\partial^{a} t_{a b}=0 \text {. }
$$

Let $t_{\rho \rho}=t_{1}$ and $t_{\rho z}=t_{2}$, then equation (118) is given by

$$
\partial_{1} t_{1}+\partial_{2} t_{2}=0, \quad \partial_{1} t_{2}-\partial_{2} t_{1}=0 .
$$

These are the Cauchy-Riemann equation for a complex function $f=t_{1}+\mathrm{i} t_{2}$. That is, a non-trivial solution of (119) implies that $f$ is an entire function on the complex plane. But $t_{1}$ and $t_{2}$ decay to zero at infinity. Hence, by Liouville's theorem, $f$ vanishes. And then, we obtain that $\beta$ is also a solution of the gauge equation (48).

Solutions of equation (92) can be obtained by other methods, for example see proposition 2.6 in [14]. In the proof above we have used the Green function in order to prove (88). It is likely that also this kind of expansion can be obtained by other methods like those used in [13]. 
To prove the mass conservation we will make use of the evolution equation given by the trace of (24), namely

$$
2 \dot{u}=-\alpha \chi+D_{a} \beta^{a} .
$$

In terms of partial derivatives this equation is written like

$$
2 \dot{u}=-\alpha \chi+\partial_{i} \beta^{i}+2 \beta^{i} \partial_{i} u \text {. }
$$

Theorem 5.3 (Mass conservation). We have

$$
\frac{\mathrm{d} m(t)}{\mathrm{d} t}=0
$$

Proof. We use equation (75) to get

$$
\frac{\mathrm{d} m(t)}{\mathrm{d} t}=\frac{1}{16} \lim _{r \rightarrow \infty} \int_{0}^{\pi}\left(\frac{1}{2} \partial_{r} \dot{\sigma}+\frac{\dot{\sigma}}{2 r}+\partial_{r} \dot{u}-\frac{\dot{u}}{r}\right) r \rho \mathrm{d} \theta .
$$

In order to prove that this integral is zero we have to compute the decay of the integrand at infinity. We begin with the terms containing $\dot{\sigma}$.

We use the relation

$$
\frac{\lambda^{\prime}}{\lambda}=\frac{\sigma^{\prime}}{2}+\frac{\rho^{\prime}}{\rho},
$$

and

$$
\rho^{\prime}=-\frac{\beta^{\rho}}{\alpha}, \quad \sigma^{\prime}=\frac{1}{\alpha}\left(\dot{\sigma}-\beta^{i} \partial_{i} \sigma\right)
$$

to conclude that

$$
\dot{\sigma}=2 \alpha \frac{\lambda^{\prime}}{\lambda}+\beta^{i} \partial_{i} \sigma+2 \frac{\beta^{\rho}}{\rho} .
$$

Using equations (55) and (88) we obtain

$$
\dot{\sigma}=o_{\infty}\left(r^{-3 / 2}\right) \text {. }
$$

For $\dot{u}$ we use equation (121) and the decay on $\beta$ to get

$$
\dot{u}=o_{\infty}\left(r^{-3 / 2}\right) \text {. }
$$

Using (127) and (128) we obtain that the boundary integral (123) vanishes.

We mention also another consequence of equation (121). From this equation we deduce that

$$
\dot{q}=o_{\infty}\left(r^{-3 / 2}\right) .
$$

Then, if the function $q$ has initially the stronger decay (76), this decay will be preserved by the evolution.

\section{Final comments}

In this final section we discuss the implication of the results presented here for the evolution of axially symmetric isolated systems.

The first question one needs to face in the evolution problem is the choice of gauge. In axial symmetry there have been studied different kind of gauge conditions (see for example 
$[2,29])$. The mass conservation formula single out a particular one. If we define appropriate Sobolev norms, then the mass formula essentially implies that the $H^{1}$ norm of the metric and the $L^{2}$ norm of the extrinsic curvature are bounded along the evolution. This is a very desirable property which is not present in other gauges. The mass conservation formula strongly suggests that the most convenient gauge for the axially symmetric evolution problem is the maximal isothermal one.

As was mentioned in the introduction, the conservation of mass is closely related with the Hamiltonian formulation of general relativity. The maximal-isothermal gauge is a dynamical gauge that depends on the time coordinate. The analysis of time-dependent gauge conditions and its relation with conserved quantities in the Hamiltonian formulation was recently studied in $[31,32]$. The maximal-isothermal gauge satisfies the fall-off conditions described in these references.

In axial symmetry, even when the gauge conditions are fixed there exist many possibilities to extract from Einstein equations a set of evolution equations. The reason for this ambiguity is that Einstein equations in three dimensions have no dynamics, this essentially means that equation (20) can be replaced by the constraint equations (26)-(27) (see [12]). If we chose to do so, then we obtain an evolution scheme in which the evolution equations are given by (18) and (19), and the other equations (including gauge conditions) are elliptic constraint. But there exist other alternatives. Following [27], we can classify them by the number of evolution equations used versus the number of elliptic constraint equations. The scheme presented above has the minimum number of evolution equations and the maximum of constraint equations. This is called a fully constrained system. On the opposite side we have a system in which we do not solve for the constraints and we solve the evolution equations (24) and (25). This is called a free evolution scheme. In between we have other possibilities to construct partially constrained systems. In all cases, the gauge conditions are solved as elliptic constraints. That is, all schemes have a mix of evolution and elliptic equations (see the discussion in chapter 3 of [27]).

To prove the mass conservation we have used two equations: the Hamiltonian constraint (26) and the evolution equation for the conformal factor of the two-dimensional metric (120). In a free evolution scheme the Hamiltonian constraint is not solved. Hence, the mass will not be conserved for arbitrary data. It will be only conserved for data that satisfies the constraint equations. Then, in this case the mass integral formula will be not useful for controlling the evolution. The same will happen with partially constrained systems in which the Hamiltonian constraint is not solved or the evolution equation (120) is not used. Such systems were discussed in [27] and in [8] (in this reference, these kinds of systems appear when the evolution of the conformal factor is used instead of the Hamiltonian constraint).

On the other hand, for a fully constrained scheme (like the ones used in $[8,28]$ ) and a partially constrained scheme in which both the Hamiltonian and the evolution equation for the metric are used (like the one studied in [17]) the mass will be conserved. The mass conservation formula singles out these two schemes. The natural problem now is to study their well posedness.

Finally, it is interesting to note that the integral mass formula has a direct application in numerical simulations. In numerical simulations of isolated systems, one often uses a position-dependent resolution that is high in the central region and much lower close to the outer boundary of the computational domain. Hence computing the mass as a volume integral is more accurate than as a boundary integral. The resulting approximation to the mass is much better conserved during the evolution ${ }^{2}[26]$.

2 I thank O Rinne for pointing this out to me. 


\section{Acknowledgments}

It is a pleasure to thank Piotr Chruściel, Robert Geroch and Martin Reiris for discussions. Special thanks to Oliver Rinne for discussions and for sharing his numerical results on a comparison of the two different ways of computing the mass. The author is supported by CONICET (Argentina). This work was supported in part by grant PIP 6354/05 of CONICET (Argentina), grant 05/B270 of Secyt-UNC (Argentina) and the Partner Group grant of the Max Planck Institute for Gravitational Physics, Albert-Einstein-Institute (Germany).

\section{References}

[1] Arnowitt R, Deser S and Misner C W 1962 The dynamics of general relativity Gravitation: An Introduction to Current Research ed L Witten (New York: Wiley) pp 227-65 (Preprint gr-qc/0405109)

[2] Bardeen J M and Piran T 1983 General relativistic axisymmetric rotating systems: coordinates and equations Phys. Rep. 96 205-50

[3] Bartnik R 1986 The mass of an asymptotically flat manifold Comm. Pure App. Math. 39 661-93

[4] Beig R and Murchadha N O 1987 The Poincaré group as the symmetry group of canonical general relativity Ann. Phys. 174 463-98

[5] Bičák J and Pravdová A 1998 Symmetries of asymptotically flat electrovacuum spacetimes and radiation J. Math. Phys. 39 6011-39

[6] Brill D 1959 On the positive definite mass of the Bondi-Weber-Wheeler time-symmetric gravitational waves Ann. Phys. 7 466-83

[7] Cantor M 1981 Elliptic operators and the decomposition of tensor fields Bull. Am. Math. Soc. 5 235-62

[8] Choptuik M W, Hirschmann E W, Liebling S L and Pretorius F 2003 An axisymmetric gravitational collapse code Class. Quantum Grav. 20 1857-78 (Preprint gr-qc/0301006)

[9] Choquet-Bruhat Y 2004 Future complete U(1) symmetric Einsteinian spacetimes, the unpolarized case The Einstein Equations and the Large Scale Behavior of Gravitational Fields (Basel: Birkhäuser) pp 251-98

[10] Choquet-Bruhat Y and Christodoulou D 1981 Elliptic systems in $H_{s, \delta}$ spaces on manifolds which are Euclidean at infinity Acta Math. 146 129-50

[11] Choquet-Bruhat Y, Isenberg J and York J W Jr 1999 Einstein constraint on asymptotically Euclidean manifolds Phys. Rev. D 61084034 (Preprint gr-qc/9906095)

[12] Choquet-Bruhat Y and Moncrief V 2001 Future global in time Einsteinian spacetimes with U(1) isometry group Ann. Inst. Henri Poincaré 2 1007-64

[13] Chruściel P T 1990 Asymptotic estimates in weighted Hölder spaces for a class of elliptic scale-covariant second order operators Ann. Fac. Sci. Toulouse Math. (5) 11 21-37

[14] Chruściel P T 2007 Mass and angular-momentum inequalities for axi-symmetric initial data sets: I. Positivity of mass Preprint arXiv:0710.3680

[15] Dain S 2007 The inequality between mass and angular momentum for axially symmetric black holes Preprint arXiv:0707.3118

[16] Dain S 2008 Proof of the angular momentum-mass inequality for axisymmetric black holes J. Diff. Geom. 79 33-67 (Preprint gr-qc/0606105)

[17] Garfinkle D and Duncan G C 2001 Numerical evolution of Brill waves Phys. Rev. D 63044011 (Preprint gr-qc/0006073)

[18] Geroch R 1971 A method for generating solutions of Einstein's equations J. Math. Phys. 12 918-24

[19] Gibbons G W and Holzegel G 2006 The positive mass and isoperimetric inequalities for axisymmetric black holes in four and five dimensions Class. Quantum Grav. 23 6459-78 (Preprint gr-qc/0606116)

[20] Gradshteyn I S and Ryzhik I M 1980 Table of Integrals, Series, and Products (New York: Academic)

[21] Maxwell D 2006 Rough solutions of the Einstein constraint equations J. Reine Angew. Math. 590 1-29 (Preprint gr-qc/0405088)

[22] Moncrief V 2006 private communication

[23] Nakamura T, Oohara K and Kojima Y 1987 General relativistic collapse to black holes and gravitational waves from black holes Prog. Theor. Phys. Suppl. 90 1-218

[24] Regge T and Teitelboim C 1974 Role of surface integrals in the Hamiltonian formulation of general relativity Ann. Phys. 88286

[25] Rendall A D 2005 Theorems on existence and global dynamics for the Einstein equations Living Rev. Relativ. 86 
[26] Rinne O 2008 private communication

[27] Rinne O 2005 Axisymmetric numerical relativity Preprint gr-qc/0601064

[28] Rinne O 2008 Constrained evolution in axisymmetry and the gravitational collapse of prolate Brill waves Preprint arXiv:0802.3791

[29] Rinne O and Stewart J M 2005 A strongly hyperbolic and regular reduction of Einstein's equations for axisymmetric spacetimes Class. Quantum Grav. 22 1143-66 (Preprint gr-qc/0502037)

[30] Ruiz M, Alcubierre M and Nunez D 2008 Regularization of spherical and axisymmetric evolution codes in numerical relativity Gen. Rel. Grav. 40 159-82 (Preprint arXiv:0706.0923)

[31] Szabados L B 2003 On the roots of the Poincaré structure of asymptotically flat spacetimes Class. Quantum. Grav. $202627-61$

[32] Szabados L B 2006 The Poincaré structure and the centre-of-mass of asymptotically flat spacetimes Analytical and Numerical Approaches to Mathematical Relativity (Lecture Notes in Phys. vol 692) (Berlin: Springer) pp 157-84

[33] Wald R M 1984 General Relativity (Chicago, IL: The University of Chicago Press) 